\title{
Computational fluid dynamics modelling to design and optimise power kites for renewable power generation.
}

PEGG, C., SURI, Y., ISLAM, S.Z., ASTHANA, A. and HOSSAIN, M. 


\section{Computational Fluid Dynamics Modelling to design and optimise Power Kites for Renewable Power Generation}

Christopher Pegg

School of Engineering, Robert Gordon University, Aberdeen, U.K.

Email: c.pegg@rgu.ac.uk

Yatin Suri*

School of Engineering, Robert Gordon University, Aberdeen, U.K.

Email:y.suri@rgu.ac.uk

*Corresponding author

Sheikh Zahidul Islam

School of Engineering, Robert Gordon University, Aberdeen, U.K.

Email: s.z.islam1@rgu.ac.uk

Abhishek Asthana

Department of Engineering and Mathematics, Sheffield Hallam University, Sheffield, U.K.

Email: a.asthana@shu.ac.uk

Mamdud Hossain

School of Engineering, Robert Gordon University, Aberdeen, U.K.

Email:m.hossain@rgu.ac.uk

Biographical notes: Christopher Pegg graduated with a MEng degree from the Robert Gordon University, Aberdeen, U.K. Christopher is currently working as a Mechanical Engineer with Optimus Aberdeen Ltd. He has a keen interest in CFD modelling of fluid flow in wind turbines and finite element analysis. He is highly motivated to apply the skills gained from the Master's degree into industrial applications.

Yatin Suri is a $\mathrm{PhD}$ graduate from the Robert Gordon University, Aberdeen, U.K. and is currently working as a Petroleum Reservoir Engineer. Before pursuing $\mathrm{PhD}$, Yatin worked as a Petroleum Engineer in the oil industry for five years. He was the top winner of Society of Petroleum Engineers (SPE) Bursary award 2017-18 and 2018-19. He graduated with a Master's degree from Institut Français du Pétrole (IFP) School, Paris, France and was awarded a gold medal for his outstanding academic performance during his undergraduate study from Indian Institute of Technology (Indian School of Mines) Dhanbad, India. His research interest includes CFD application in renewable energy and petroleum engineering.

Sheikh Zahidul Islam is Academic Strategic Lead (Head of Mechanical Engineering Discipline) with key expertise in Computational Fluid Dynamics (CFD) simulations and mathematical modelling. His research interests include PEM fuel cell as an energy storage device, CFD modelling of multi-phase flows, CFD modelling of wind turbine $\&$ heat transfer from finned tube banks, Thermosyphon \& fluidized bed heat exchanger for industrial application. Sheikh is involved in applying CFD techniques and optimization to solve some of the most challenging and interesting problems related to emerging energy sources such as fuel cell and wind/ tidal turbine. 
Abhishek Asthana is the Director of Hallam Energy, the energy research group at Sheffield Hallam University (SHU). In 2009, he co-founded Hallam Energy and has since led and delivered more than 55 projects of industrial energy research, consultancy and knowledge transfer. He has won $£ 9.5$ million funding, authored 37 scientific papers and 2 books, invented 4 patents and developed 5 commercial software packages. He is the deputy director of the Doctoral Training Alliance (DTA) in Energy which won winning €6.5 Million funding from the European Commission and consists of $90 \mathrm{PhD}$ students and 180 Supervisors across 19 British Universities.

Mamdud Hossain is a Reader in Thermofluids at the School of Engineering of Robert Gordon University. He leads the Flow Modelling Research Group. His research interests include applying computational modelling techniques to solve some of the most challenging and interesting problems related to the hydrogen fuel cell, combustion and multiphase flows for oil and gas industry. His research has been funded through the Northern Research Partnership, Innovate UK, OGIC and the Carnegie Trust. He is a Science Board member of EPSRC Hydrogen and Fuel Cell Supergen Hub. He is Chartered Engineering and member of IMechE and a Senior Fellow of the Higher Education Academy.

\begin{abstract}
Power kites provide the potential rewards of obtaining the disused energy supply from high altitude wind. This paper aims to provide a design of Power kite and optimise the potential for renewable power generation. The Power kite was modelled using Computational Fluid Dynamics to study its characteristics. The numerical modelling results were compared against the wind tunnel experimental study and two 3D printed Power kites. The design was optimised using several variables, of which include aerofoil choice, surface roughness, wind speed and operating parameters. The results suggest that operating the kites at minimum $15 \mathrm{~m}$ horizontal separation is favourable, with the trailing kite operating below the leading, removing the potential for this kite to operate in the wake turbulence of the first. This paper presents relevant, applicable data which can be used for predicting the performance, and potentially optimising further Power kite design.
\end{abstract}

Keywords: Power kites, Computational fluid dynamics, Wind energy, Renewable power generation, Surface roughness, Aerofoil, Kite optimisation, Billows, Drag coefficient, Lift coefficient

\title{
1. Introduction
}

Wind energy is the world's fastest-growing renewable resource sector, and in many countries, is the largest provider of renewable power (Blanco, 2009). Unfortunately, wind turbines have inherent limitations when harnessing the power of the wind. It is well recognised that with increased altitude, it results in higher wind power densities, and this is reflected in the continual rise in wind turbine height (Zhang, 2013). The current wind turbine technology possesses limitation to harness the abundant natural resource of high-altitude wind (Canale, Fagiano \& Milanese, 2009). Power kites are more effective at high altitudes due to both aerofoil design and location of components (Gerakopulos, Boutilier \& Yarusevych, 2010), the generator of a power kite is left on the ground; connected by a tether. This allows the kite to spin the generator, as the 
available power at high altitudes is significantly greater (Zhang, 2013, Costello et al., 2015).

Being a somewhat novel approach, there exist few readily available resources to accurately predict kite performance (Kheiri et al., 2018, Stevenson, Alexander \& Lynn, 2005). Without accurate performance prediction and the ability to optimise kite design at an early stage, these power generation systems will struggle to reach their full potential (Bauer et al., 2018). Thus, it is essential to maximise the lift produced to optimise the efficiency. Previous research (Canale, Fagiano \& Milanese, 2009, Argatov, Rautakorpi \& Silvennoinen, 2011, Zhang, 2013, Bauer et al., 2018) has focussed on improving the aerofoil shape. While this is one of the critical parameters in improving the efficiency of power kites, surface roughness has been neglected in these studies. An aerofoil with a high surface roughness will produce turbulent airflow over the body, which will, in turn, increase the resistance due to drag on the kite (Fechner et al., 2015, Castelino, Jana, 2018). Drag also occurs on the tether over its length. Over 500 metres, it is possible to develop significant drag, enough to have a large impact on the overall efficiency of the system(Argatov, Rautakorpi \& Silvennoinen, 2011). It is, therefore, necessary to study aerodynamic drag over the kite, while also optimising the aerofoil shape to produce maximum lift (Canale, Fagiano \& Milanese, 2009, Zhang, 2013, Gaunaa et al., 2011). This paper uses computational fluid dynamics to solve Navier-Stokes equations and model the wind flow in design and optimisation of power kite, under varying input parameters, namely horizontal and vertical separation distance and surface roughness. The impact of the change in lift and drag coefficients has been investigated in detail.

Over the past several years, the aerodynamic performance of kites has improved dramatically (Cherubini et al., 2015). This performance increase is mainly due to the increased popularity of kite related sports, i.e. kite surfing, kiteboarding, and paragliding (ISAF Kiteboarding, 2012), the latter being essentially a form of an untethered kite. Due to the increased interest and therefore, market, significant effort has gone into improving efficiency and controllability (Blanco, 2009). There are many variations within kite design, but the highest proportion of kites within this sector, fall into two categories, namely, foil kites and leading edge inflatable kites (Houghton, Carpenter, 2003)

\subsection{Kite Aerodynamic principles}

The basic geometry of an aerofoil is shown in Figure 1. The size of the kite/wing is principally defined by its chord length $\left(\mathrm{L}_{\mathrm{c}}\right)$ and span (b). The chord (c) of an aerofoil refers to an imaginary straight line joining the leading and trailing edges, and the chord length, as its name suggests, is the length of the chord. The second principal measurement is the span (b). As a kite has a specific arc, the span must be further defined. When referring to a kite, there are two terms that are commonly used, the first is projected span $\left(b_{p}\right)$, and the second is flat span (b). Projected span is illustrated in Figure 2.

Both the chord length and the span, whether projected or flat, are used to find a critical definition of both kites and wings. This definition is referred to as Aspect Ratio (AR), depending upon which span is used, the aspect ratio can be either flat or projected. The aspect ratio is the ratio of the span (b) to the chord (c) (Schmehl, 2018). A high aspect ratio indicates long, narrow wings, whereas a low aspect ratio indicates short, stubby wings. As most wings and kites are lofted, meaning that the chord is not constant but 
varies along the wing, so the aspect ratio is usually defined as the square of the span (b), divided by the area (A) of the wing planform (Schmehl, 2018).

$$
A R=\frac{b^{2}}{A}
$$

The next fundamental parameter of an aerofoil is the Angle of Attack (AoA) denoted by $\alpha$. This is defined in aerodynamics as the angle between the chord line and the vector representing the relative motion between the fluid and kite (Houghton, Carpenter, 2003). Increased AoA is associated with increasing lift coefficient until it reaches its maximum and starts to decrease.

\subsection{Lift and Drag Coefficients}

All wings work on the basic principle of a difference in pressure across the upper and lower surfaces (Low Pressure on the Upper Surface, High Pressure on the Lower) creating a pressure gradient and thus a lift force. This force is dependent on four factors - Lift Coefficient, Fluid Density, Wing Velocity and the Wing Area - as shown in the equation (2) (Houghton, Carpenter, 2003, Schmehl, 2018)

$$
F_{l}=\frac{1}{2} \rho A_{p} V_{A}^{2} C_{l}
$$

As both the fluid density and velocity are parameters surrounding the aerofoil, the lift of the wing is maximised by maximising the lift coefficient and the surface area. A drawback of indefinitely increasing the surface area of the wing is both the weight and the drag coefficient will increase. As the weight will begin to increase exponentially over a very large surface area for a minimal return, it is assumed that the best method for maximising the lift is to maximise the lift coefficient.

The Lift and Drag Coefficients are unitless, dimensionless parameters that relate to the lift and drag of a wing, respectively. The lift coefficient refers to the dynamic pressure multiplied by the surface area of the wing represented as a ratio; the lift coefficient can vary depending on the AoA that the wing is operating. As the AoA rises, the coefficient of lift rises until the maximum for each respective aerofoil is reached, this is known as the 'stall point' of the aerofoil, and an increase of AoA will reduce the lift coefficient. The Drag coefficient is a parameter that defines the total force resisting the wings movement through a fluid, by both the frictional forces associated with the fluid flowing past the wing surface and a localised high pressure at the front of the wing resisting the forward momentum of the kite. This coefficient can be determined as shown in equation (4) (Houghton, Carpenter, 2003, Schmehl, 2018)

$$
\begin{gathered}
F_{d}=\frac{1}{2} \rho A_{p} V_{A}^{2} C_{d} \\
C_{d}=\frac{F_{d}}{\frac{1}{2} \rho A_{p} V_{A}^{2}}
\end{gathered}
$$

The net force is a vector summation of the lift force $\left(F_{l}\right)$ and the drag force $\left(F_{d}\right)$.

$$
\overline{F_{n}}=\overline{F_{l}}+\overline{F_{d}}
$$

The coefficient, as shown in equation (4) shows that the most straightforward way of reducing the drag coefficient is to either minimise the drag force or maximise the nominal area of the wing. In the aerospace industry, the nominal area is taken as the 
surface area of the wing. The drag force of the aerofoil can be reduced by improving the surface finish; rough surfaces can cause turbulence- known as the vorticity- that increases the resistive forces acting upon the wing (Gohl, Luchsinger, 2013).

The remaining properties of the aerofoil shown in Figure 1, are dependent upon the geometry of the aerofoil. These geometric properties are important as they define the location of the point where the relative forces act. For instance, the Centre of Mass $(\mathrm{CoM})$ is a point related to the physical dimensions of the aerofoil and is the location where mass $(\mathrm{m})$ and gravity $(\mathrm{g})$ act through. Compared to the $\mathrm{CoM}$, the Center of Pressure $(\mathrm{CoP})$ is not a fixed point. Its relative position on the chord (c), is determined by the sum of pressure force exerted on the surface of the aerofoil (Houghton, Carpenter, 2003). These pressure forces are not fixed; they change with the AoA and the fluid flow velocity.

Due to the variable nature of the CoP, and the difficulty in determining its exact position, engineers have defined a third point which is used to calculate the aerodynamic forces. This point is defined as a quarter chord (c/4), referenced from the leading edge. It has been found both experimentally and analytically that, if the aerodynamic force is applied at a $\frac{1}{4}$ chord $\left(\frac{c}{4}\right)$, the magnitude of the aerodynamic moment $\left(F_{m}\right)$ remains almost constant with the angle of attack $(\alpha)$ (Schmehl, 2018).

$$
F_{m}=\frac{1}{2} \rho A_{p} V_{A}^{2} C_{m}
$$

In order to define efficiency for a 2D aerofoil, the standard method is to use the ratio of Lift force to Drag force; this is expressed in equation (7).

$$
\eta=\frac{F_{l}}{F_{d}}=\frac{C_{l}}{C_{d}}
$$

\subsection{Powerkite Design}

Multiple potential designs of powerkite are available for optimisation; variations include the kite's size, choice of the aerofoil, dimensions and whether billows are added. Due to the low wind speed available, while these kites are in use, the most practical aerofoil design is symmetrical in shape, due to the low speed and Reynolds number; symmetrical aerofoils are more efficient in generating lift with these conditions compared to cambered aerofoils (Matsson et al., 2016). In the present study, NACA 0018 Aerofoil is used (Gerakopulos, Boutilier \& Yarusevych, 2010). The kite dimensions can be found in Table 1.

\section{Governing Equations}

The Power kite was modelled using Computational Fluid Dynamics (CFD) by solving the Navier-Stokes equation (continuity and momentum conservation equations) assuming isothermal, steady-state, and incompressible flow. The governing equations for the time-dependent 3-D fluid flow around a body are the continuity equation and momentum equation. As the fluid flow is considered incompressible and isothermal, therefore the continuity and momentum equations can be written in incompressible form, neglecting the density terms (Versteeg, Malalasekera, 2007). 
2.1. Continuity equation

$$
\frac{\partial u}{\partial x}+\frac{\partial v}{\partial y}+\frac{\partial w}{\partial z}=0
$$

\subsection{Momentum equation}

$$
\begin{gathered}
\rho\left(\frac{\partial u}{\partial t}+u \frac{\partial u}{\partial x}+v \frac{\partial u}{\partial y}+w \frac{\partial u}{\partial z}\right)=-\frac{\partial p}{\partial x}+\mu\left(\frac{\partial^{2} u}{\partial x^{2}}+\frac{\partial^{2} u}{\partial y^{2}}+\frac{\partial^{2} u}{\partial z^{2}}\right) \\
\rho\left(\frac{\partial v}{\partial t}+u \frac{\partial v}{\partial x}+v \frac{\partial v}{\partial y}+w \frac{\partial v}{\partial z}\right)=-\frac{\partial p}{\partial y}+\mu\left(\frac{\partial^{2} v}{\partial x^{2}}+\frac{\partial^{2} v}{\partial y^{2}}+\frac{\partial^{2} v}{\partial z^{2}}\right) \\
\rho\left(\frac{\partial w}{\partial t}+u \frac{\partial w}{\partial x}+v \frac{\partial w}{\partial y}+w \frac{\partial w}{\partial z}\right)=-\frac{\partial p}{\partial z}+\mu\left(\frac{\partial^{2} w}{\partial x^{2}}+\frac{\partial^{2} w}{\partial y^{2}}+\frac{\partial^{2} w}{\partial z^{2}}\right)
\end{gathered}
$$

The CFD code used to perform the simulation was ANSYS Fluent. The specific solver chosen was a Reynold's Averaged Navier-Stokes (RANS) method due to its inherent lower computational cost. The simulations flow was modelled using the SST k- $\omega$ model. This particular model is well established for use with external aerodynamics(Gohl, Luchsinger, 2013). It is a two-equation model and is regarded as having excellent treatment of boundary layers, especially for high adverse pressure gradients (Menter, 1993). This code employs an unstructured, collocated finite-volume technique. The convection contribution to the velocity increment is predicted by an upwind scheme, while the centred spatial discretisation of the convection is introduced as a semi-implicit pressure-correction method (SIMPLE algorithm) (Versteeg, Malalasekera, 2007).

\section{Computational Domain}

The computational domain used in the present study is in the form of a cuboid with dimensions $1.22 \mathrm{~m} \times 0.46 \mathrm{~m} \times 0.46 \mathrm{~m}$ based on the wind tunnel experiment performed shown in Figure 3. The kite is located at $0.6 \mathrm{~m}$ from the inlet. To determine the relative size of the 3-D model kite in comparison to the computational domain representing the wind tunnel test section, the blockage ratio $(\gamma)$ was calculated. This is a ratio between the frontal area of the kite model, including the support, and the cross-sectional area (CSA) of the domain representing the wind tunnel test section.

$$
\text { Blockage Ratio }(\gamma)=\frac{\text { Kite frontal area }}{\text { Tunnel CSA }}
$$

With a blockage ratio of $5 \%$, this results in a kite model with a projected span $\left(b_{p}\right)$ of $0.2 \mathrm{~m}$. With the kite model and kite prototype projected spans, it is possible to calculate the scaling factor $(\lambda)$. With such a low blockage ratio (5\%), this helps to ensure that the boundaries of the computational domain will have minimal effect on the air flow over the kite. 
A velocity inlet boundary condition was used with $10 \mathrm{~m} / \mathrm{s}$ at inlet and pressure outlet was used at the outlet with $0 \mathrm{~Pa}$ pressure. All the other boundaries were configured as a no-slip wall.

\section{Mesh Independence}

To validate the simulation results, a mesh independence study was performed. The mesh type used in the current study was a structured cut-cell mesh. Cut-cell meshes are quick to generate, tend to result in predominantly high quality hexahedral elements, and solution convergence is generally faster. A significant benefit is that they can be refined/coarsened in specific regions through the use of size-functions. The mesh independence study involved progressively refining the mesh while monitoring the lift $\left(C_{L}\right)$ and drag $\left(C_{D}\right)$ coefficients. Convergence was deemed to have been reached when refining the mesh began to have little to no effect on both these coefficients. Thus, the number of cells used in the current study are $6.5 \mathrm{e} 5$.

\section{Experimental Validation}

In order to provide validation of the CFD simulation, a direct comparison was made between the simulated results, dimensionless analysis and the experimental results obtained via low-speed wind tunnel testing (Figure 5). It can be seen from Figure 5 that the experimental lift and drag results obtained via the low-speed wind tunnel, are slightly increased compared to the simulated CFD results. The deviation in the CFD results from the experimental results can be explained by CFD model is based on the assumption of the air entering the boundary is uniform, with constant air density and perfectly smooth kite surface. However, in the experimental setup, the air density is not uniform, and the 3D models have minor roughness. Although the experimental lift and drag coefficient curve show slightly higher values than the CFD results, the curve is still a close match, i.e. stall occurs at an equivalent angle of attack of $40^{\circ}$ with a percentage error of $10 \%$ suggesting the CFD model can be used for further analysis.

\section{Results and discussion}

While in operation for power generation, the power kites will likely be operating in close proximity. A single kite can create a large area of turbulent flow, which can impact the efficiency of a kite operating nearby. To find an ideal distance that the kites can operate safely two kites were simulated by varying horizontal separation distances from $200 \mathrm{~mm}, 400 \mathrm{~mm}$ to $550 \mathrm{~mm}$ and vertical separation distance from $150 \mathrm{~mm}, 0$ $\mathrm{mm}$ to $-150 \mathrm{~mm}$. It is to be noted that the simulation is scaled using a 1:35.5 model; the distance separating the models can be scaled to appropriate the actual position when in industrial use.

Firstly, a single kite was modelled using the CFD technique described earlier to analyse the performance, and then the two kites are modelled together. The CFD results from modelling the single kite are shown in Figure 6-8. Figure 6 shows the velocity over a single aerofoil at $10 \mathrm{~m} / \mathrm{s}$ and the relevant AoA, when the kite is flying horizontally $\left(\right.$ AoA $0^{\circ}$ ) the variation in velocity is limited to the immediate surroundings of the aerofoil, this creates a low difference in pressure, which produces both a low 
Coefficient of Lift and Drag, as shown in Figure 8. As the AoA increases, the turbulence created by the kite increases, until the area of turbulent flow is reached, as shown in Figure 7. This is the maximum AoA achievable before stalling begins to impact the lift coefficient, the air velocity above the kite is shown to be very low, which is present for a significant distance from the wing itself.

As the wing is flying directly into the apparent wind at an AoA of $0^{\circ}$, it is expected that the vorticity region will be relatively small, this can be assumed due to the flow being laminar, and the kite surface assumed to be perfectly smooth. Figure 7a shows the vorticity region over the kite at this respective AoA; it is shown in this figure that the region is relatively small, with the vortex created by the wing being located mainly at either end of the kite. These are known as wingtip vorticities, where the air swirls around the end of a wing, caused by the lift generated by a wing, this phenomenon is present in all aerofoils. The vortex regions around the wingspan are significantly smaller than these wingtip vorticities and will have a minimal impact on the drag coefficient of the wing. At an AoA of $40^{\circ}$ the vortex region, as represented in Figure $7 \mathrm{~b}$, shows a large region, which coincides with the area of turbulent velocity shown within Figure. The large vortex region creates the large drag coefficient for this AoA. An increase in this angle causes the kite to stall, significantly increasing the drag coefficient.

Next to find the ideal distance the kites can operate safely two kites were simulated by varying horizontal separation distances from $200 \mathrm{~mm}, 400 \mathrm{~mm}$ to $550 \mathrm{~mm}$ and vertical separation distance from $150 \mathrm{~mm}, 0 \mathrm{~mm}$ to $-150 \mathrm{~mm}$.

Figure 9 shows the velocity streamlines over each kite, which represents the airflow over the kites under the specific conditions. From Figure 9a it can be shown that at a low AoA the airflow over each wing is uniform and laminar, it also shows that the velocity over the first wing is greater than that of the second, due to the disruption of airflow created by the first wing. This variation in velocity is matched by a difference in the size of the pressure zones. On the first kite, the areas of high and low pressure are larger than that of the second, which in turn reduces the lift coefficient of the second kite, as shown in Figure 9b.

At an AoA of $40^{\circ}$, it is seen, in Figure 11a and that the airflow above the wing is turbulent, caused by the air rushing into this area of low pressure created by the wing. This occurs on both wings, with the area of low pressure above the second wing being larger and extending beyond the rear of the kite, this increases the drag ratio, as shown in the graphs in figures and where the drag coefficient for the second kite is higher than that of the first kite.

The plots in Figure 10 show the coefficient of lift and drag against the AoA. The lift and drag of the first kite is a more uniform response, gradually rising as the AoA increases. While the drag of the second kite is similar, generally slightly higher than that of the first, the lift coefficient varies significantly, at an AoA of both $20^{\circ}$ and $30^{\circ}$ the lift coefficient appears to drop before rising again. It is likely that as the flow over the first kite transitions from laminar to turbulent the air reaching the second kite is irregular in flow, thus having an impact on the efficiency of the second kite.

Figure 12a shows both the comparison of the Lift and Drag Coefficients of the 3 different simulations when comparing the Drag Coefficients, it can be concluded that the drag is not a significant factor in the choice of arrangement to be carried forward, the coefficient of lift, however, does vary, meaning a setup can be chosen from this. 
In comparison, the Lift produced by each wing is very similar throughout each simulation, with the most significant variation occurring at an AoA of both $15^{\circ}$ and $25^{\circ}$. At these deviations, the lowest Lift Coefficient, and therefore the lowest efficiency, is seen to be at a horizontal displacement of $400 \mathrm{~mm}$, in this setup, the leading kite produces the lowest lift coefficient out of the three simulations, with the rear producing the 2 nd lowest. From these results, it can be concluded that the horizontal displacement of $400 \mathrm{~mm}$ creates the least efficient setup.

From the Figure $12 \mathrm{~b}$ of vertical separation case, the drag coefficient is almost identical for each setup, the only variation being at $15^{\circ}$ AoA for the trailing kite located above the leading. The lift coefficient for each kite working close together is greater than that of the single for most AoA. The most significant lift coefficient, produced at $40^{\circ}$, is created by the leading kites in both setups, the trailing kite located $150 \mathrm{~mm}$ below generally produces a larger lift over a wider AoA. Therefore, this setup will be able to obtain more energy out of the wind, thus obtaining a more efficient setup for power generation.

\subsection{Increase in wind speed}

While it is known that the global average wind speed is $7.2 \mathrm{~m} / \mathrm{s}$ at an altitude of $800 \mathrm{~m}$, it is possible for larger gusts to occur, because of this it is necessary to simulate the kite at higher velocities to ensure an effective method is in place to obtain the energy from this wind. To have an improved understanding of the performance of the power kites at higher wind speed, a further investigation was carried out by increasing the wind speed from $10 \mathrm{~m} / \mathrm{s}$ to $15 \mathrm{~m} / \mathrm{s}$. validate the results generated at a wind speed of $10 \mathrm{~m} / \mathrm{s}$. Figure 13 shows the lift and drag coefficients at a windspeed of both $10 \mathrm{~m} / \mathrm{s}$ and $15 \mathrm{~m} / \mathrm{s}$.

Figure 13a-b show the Lift and Drag Coefficients of the aerofoils at a separation of 150 $\mathrm{mm}$ vertically, with Figure 13c-d a horizontal separation of $400 \mathrm{~mm}$. The Drag coefficients produced by each wing are near identical at each respective wind speed, with the drag produced at $15 \mathrm{~m} / \mathrm{s}$ generally being slightly larger than that of the slower speed, caused by ANSYS assuming fully developed laminar flow.

The lift coefficient shows the greatest change between the two airspeeds, with the coefficient at least doubling in magnitude for all AoA. This is generated due to a larger pressure difference created over the aerofoil, as shown in Figure 14, with the same scale applied for each figure, the high pressure on the bottom of the wing is a lot greater at $15 \mathrm{~m} / \mathrm{s}$ compared to $10 \mathrm{~m} / \mathrm{s}$, and the low pressure on the top is the same, respectively. From this, it can be deduced that a kite operating at a lower wind speed will produce a lower lift coefficient, while the drag will remain relatively similar to previous experiments.

\subsection{Effect of Surface Roughness}

All previous CFD results assume the surface of the kite to be perfectly smooth. In reality, the surface roughness is present and play an essential role. Therefore, simulating a surface roughness on the kite is an essential factor. At an altitude of $800 \mathrm{~m}$ (Roughly 2600 feet), the average air temperature is slightly under $10^{\circ} \mathrm{C}$ (Menne et al., 2012), the combination of this and the windchill factor can create situations where the temperature nears freezing, at these low temperatures frost can form on the kite surface, this can create roughness on the surface, while minor, it will have an impact on the efficiency of the kite. The roughness height created by frost is roughly $0.5 \mathrm{~mm}$ (Kim et al., 2019). 
To account for additional surface roughness on the surface of the wing, a roughness factor of $1 \mathrm{~mm}$ was chosen for use in the current study.

The roughness height and roughness constant used in the present study is $0.001 \mathrm{~m}$ and 0.5. The results obtained are shown in Figure 15. Figure 15 compares the drag of a smooth kite with that of the rough; it is shown that the rough kite has a larger drag coefficient and a lower lift. A larger drag is expected, as the kite surface imposes a larger resistive force to the air flowing over it, which in turn reduces the wind speed over the kite surface and reduces the lift.

Figure 16a shows the region of vorticity around the kite, one of the notable changes between this and the smooth kite is the vorticity on the kite surface, on the smooth kite the vortex regions on the surface are minor, while the rough kite has a larger region, most notably the wingtip vortices, this, in turn, leaves a large vortex region in the kite's wake. Figure 16b shows the velocity over the kite; the boundary layer thickness over the surface has increased due to the addition of surface roughness. This larger boundary increases the area of flow velocity on the kite surface, which is a contributing factor in the increased vortex region of the rough kite, the larger boundary layer can reduce the pressure difference over the aerofoil, which can, in turn, reduce the lift coefficient.

\subsection{Kite Optimisation}

Optimisation of the kite is an essential factor in the design, as the renewable energy production is solely dependent on the aerodynamic efficiency of the kite. Some of the kite factors investigated in the current study are as follows:

- Whether Billows improve or reduce the Lift Coefficient

- How changing the Aerofoil used in the design may impact efficiency

By maximising the possible energy production per kite, the initial costs for building the unit can be reduced (Selecting Cheaper Materials, using a wing shape that is easier to replicate and simply reducing the number of units required per system).

\subsubsection{Billows}

A billowed kite requires flowing air to inflate the kite to the desired shape, thus allowing the lift to be generated. This method is cost-reducing as it removes the requirement for a solid structure to maintain the aerofoil shape regularly. It also reduces the risk of damages to property and persons by reducing weight and allowing the kite to collapse under itself should the tether break during operation (Houghton, Carpenter, 2003).

The billows were added to the design by adding equation driven curves to the unextruded outline of the wing, by mirroring these along the Z-Plane this ensured an equal billow on the top and bottom surface of the wing. A completed model of the billowed wing can be found in Figure 17, with the un-billowed wing used for reference.

Following re-meshing and application of necessary boundary conditions, the billowed kite was simulated at speeds of 10 , and $15 \mathrm{~m} / \mathrm{s}$, a sample of the results can be found in Figure 18. The lift and drag coefficients are plotted against that of an un-billowed kite for comparison.

From Figure 18 it is clearly shown that the Drag of the billowed kite is greater than that of the un-billowed, created by both an increase in drag force and a change in surface area - Drag Coefficient is inversely proportional to the surface area. The plot maintains 
a similar gradient between both windspeeds, only experiencing a change in magnitude, showing a pattern in the drag coefficient at varying wind speeds.

The lift follows a similar pattern as observed in the drag, with the gradient of the plot being maintained between speeds. Generally, the lift produced by the billowed kite is less than the un-billowed variant. The exception being at high attack angles at $15 \mathrm{~m} / \mathrm{s}$ where the billowed kite produces greater lift, while this may be advantageous in specific applications it is not useful for power kites, due to the average wind speed at the height of $800 \mathrm{~m}$, this windspeed is seldom reached, and therefore using this design will reduce the maximum efficiency of the system. While this may be disadvantageous in maximising energy production, a positive lift is available at all AoA, leaving a potential for re-design, increasing the lift above that of the un-billowed kite and potentially increasing the overall efficiency of the system.

\subsubsection{Aerofoil}

Another method of optimisation of the power kite is to change the aerofoil of which the kite is based off. A comparison is made between two different aerofoils, namely NACA 0018 (Gerakopulos, Boutilier \& Yarusevych, 2010) and NACA 2412 (Matsson et al., 2016) based on the availability of the co-ordinates of the 2412 and 0018 . The NACA 0018 aerofoil is known as a symmetrical aerofoil, where the chord line is straight and acts as the plane of symmetry for the top and bottom surface, whereas 2412 is known as a cambered aerofoil, where the chord line is curved and does not represent a line of symmetry. Figure 19 shows the cross-section of the NACA 0018 and 2412 aerofoil, respectively.

The 2412 aerofoil was substituted into the power kite design, this was then tested at both 10 , and $15 \mathrm{~m} / \mathrm{s}$, plots of the lift and drag at these conditions are found in Figure 20 .

As seen in Figure 20 the NACA 2412 Aerofoil produces a larger Lift than the 0018; the increase is most significant at $15 \mathrm{~m} / \mathrm{s}$, where the maximum lift is roughly 1.75 times greater than that of the 0018 . The drag produced at each wind speed is relatively similar, as expected the drag at $15 \mathrm{~m} / \mathrm{s}$ is greater than the lower speed. The trend available from this data is that the lift produced from the 2412 aerofoil increases at a greater rate at higher speeds when compared to 0018 . As the average wind speed is less than $10 \mathrm{~m} / \mathrm{s}$, averaging at $7.2 \mathrm{~m} / \mathrm{s}$ at $800 \mathrm{~m}$ altitude, if the trend is equal and opposite at slower speeds, that will imply the lift generated would be less than the 0018 at these lower speeds. Reducing the efficiency of the system and reducing the economic viability of the setup. From this, the most suitable aerofoil and kite shape to be used to produce power kites are the NACA 0018 Aerofoil, with a fixed, un-billowed elliptical shape.

\section{Conclusion}

This paper aimed to optimise a power kite suitable for renewable power generation; one of the main topics discussed was the operating distance between kites and how it impacts the efficiency of each kite. Generally, the leading kite is not impacted when working in close vicinity to other kites, the trailing one, however, was subject to large variations in lift and drag coefficient. These results suggested that the optimal positions for the rear kite are either to be directly behind the leading kite, a large distance between the two (at least $15 \mathrm{~m}$ ) or for the trailing kite to operate below the leading. Due to the 
risk of kite tethers wrapping around each other if operating in close proximity, the ideal choice is operating the kites at minimum $15 \mathrm{~m}$ horizontal separation, with the trailing kite operating below the leading, removing the potential for this kite to operate in the wake turbulence of the first. As the wind speed is generally very low at low altitudes, it is recommended to use a symmetrical aerofoil for the kite design, as these tend to produce greater lift with low Reynolds airflow.

\section{Acknowledgement}

This research is supported by the School of Engineering, Robert Gordon University, Aberdeen, United Kingdom.

\section{Conflicts of Interest}

The authors declare no conflicts of interest.

\section{References}

Argatov, I., Rautakorpi, P. \& Silvennoinen, R. 2011, "Apparent wind load effects on the tether of a kite power generator", Journal of Wind Engineering and Industrial Aerodynamics, vol. 99, no. 10, pp. 1079-1088.

Bauer, F., Kennel, R.M., Hackl, C.M., Campagnolo, F., Patt, M. \& Schmehl, R. 2018, "Drag power kite with very high lift coefficient", Renewable Energy, vol. 118, pp. 290-305.

Blanco, M.I. 2009, "The economics of wind energy", Renewable and sustainable energy reviews, vol. 13, no. 6-7, pp. 1372-1382.

Canale, M., Fagiano, L. \& Milanese, M. 2009, "High altitude wind energy generation using controlled power kites", IEEE Transactions on Control Systems Technology, vol. 18, no. 2, pp. 279-293.

Castelino, R.V. \& Jana, S. 2018, "Analytical and Numerical computational experiment to perceive the response of high altitude Wind energy system using Kite", 2018 4th International Conference on Electrical Energy Systems (ICEES)IEEE, , pp. 381.

Cherubini, A., Papini, A., Vertechy, R. \& Fontana, M. 2015, "Airborne Wind Energy Systems: A review of the technologies", Renewable and Sustainable Energy Reviews, vol. 51, pp. 1461-1476.

Costello, S., Costello, C., François, G. \& Bonvin, D. 2015, "Analysis of the maximum efficiency of kite-power systems", Journal of renewable and sustainable energy, vol. 7, no. 5, pp. 053108 .

Fechner, U., van der Vlugt, R., Schreuder, E. \& Schmehl, R. 2015, "Dynamic model of a pumping kite power system", Renewable Energy, vol. 83, pp. 705-716.

Gaunaa, M., Carqueija, P.F.P., Réthoré, P.M. \& Sørensen, N.N. 2011, "A computationally efficient method for determining the aerodynamic performance 
of kites for wind energy applications", EWEA Annual Event 2011European Wind Energy Association (EWEA), .

Gerakopulos, R., Boutilier, M. \& Yarusevych, S. 2010, "Aerodynamic characterization of a NACA 0018 airfoil at low Reynolds numbers", 40th Fluid dynamics conference and Exhibit, pp. 4629.

Gohl, F. \& Luchsinger, R.H. 2013, "Simulation based wing design for kite power" in Airborne Wind Energy Springer, , pp. 325-338.

Houghton, E.L. \& Carpenter, P.W. 2003, Aerodynamics for engineering students, Elsevier.

Kheiri, M., Bourgault, F., Nasrabad, V.S. \& Victor, S. 2018, "On the aerodynamic performance of crosswind kite power systems", Journal of Wind Engineering and Industrial Aerodynamics, vol. 181, pp. 1-13.

Kim, J., Kwon, D., Shin, P., Baek, Y., Park, H., DeVries, K.L. \& Park, J. 2019, "Evaluation of surface roughness and frost retardancy of a glass fiber/unsaturated polyester composite", International Journal of Heat and Mass Transfer, vol. 130, pp. 282-289.

Matsson, J.E., Voth, J.A., McCain, C.A. \& McGraw, C. 2016, "Aerodynamic Performance of the NACA 2412 Airfoil at Low Reynolds Number", 2016 ASEE Annual Conference \& Exposition.

Menne, M.J., Durre, I., Vose, R.S., Gleason, B.E. \& Houston, T.G. 2012, "An overview of the global historical climatology network-daily database", Journal of Atmospheric and Oceanic Technology, vol. 29, no. 7, pp. 897-910.

Menter, F. 1993, "Zonal two equation kw turbulence models for aerodynamic flows", 23rd fluid dynamics, plasmadynamics, and lasers conference, pp. 2906.

Schmehl, R. 2018, Airborne wind energy: advances in technology development and research, Springer.

Stevenson, J., Alexander, K. \& Lynn, P. 2005, "Kite performance testing by flying in a circle", The Aeronautical Journal, vol. 109, no. 1096, pp. 269-276.

Versteeg, H.K. \& Malalasekera, W. 2007, An introduction to computational fluid dynamics: the finite volume method, Pearson Education.

Zhang, H. 2013, "Kite modeling for higher altitude wind energy", Energy and Power Engineering, vol. 5, no. 07, pp. 481. 
Table 1 Kite dimensions

\begin{tabular}{ccc}
\hline Component & Length $(\mathbf{m})$ & Angle $\left(^{\circ}\right)$ \\
\hline Wingspan & 11.128 & N/A \\
Centre Chord & 2.903 & N/A \\
End Chord & 1.7418 & N/A \\
Kite Arc & N/A & 180 \\
\hline
\end{tabular}


Figure 1 Aerofoil geometry and forces (Houghton, Carpenter, 2003)

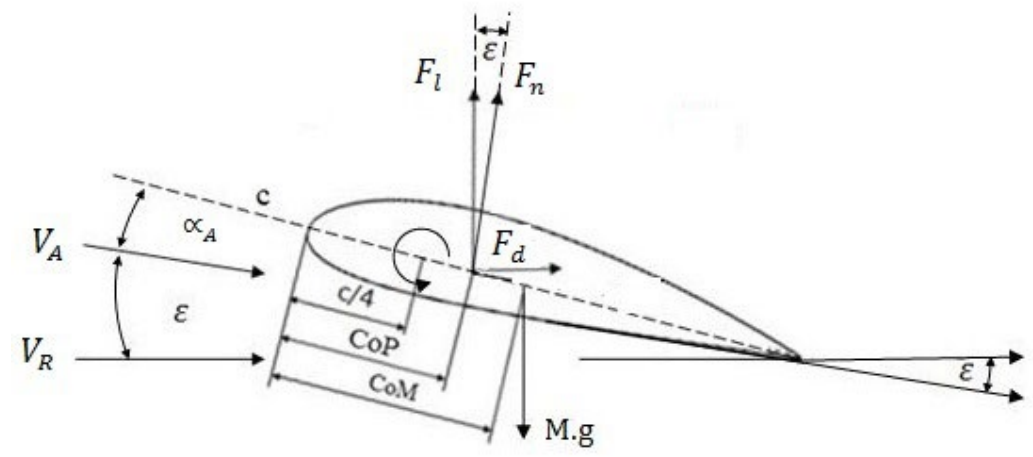

Figure 2 Projected Span

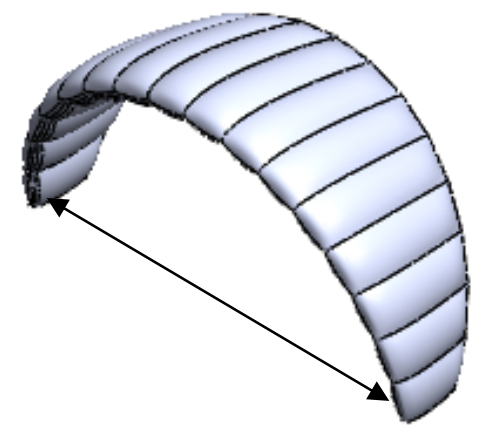

Figure 3 Computational domain, in meters

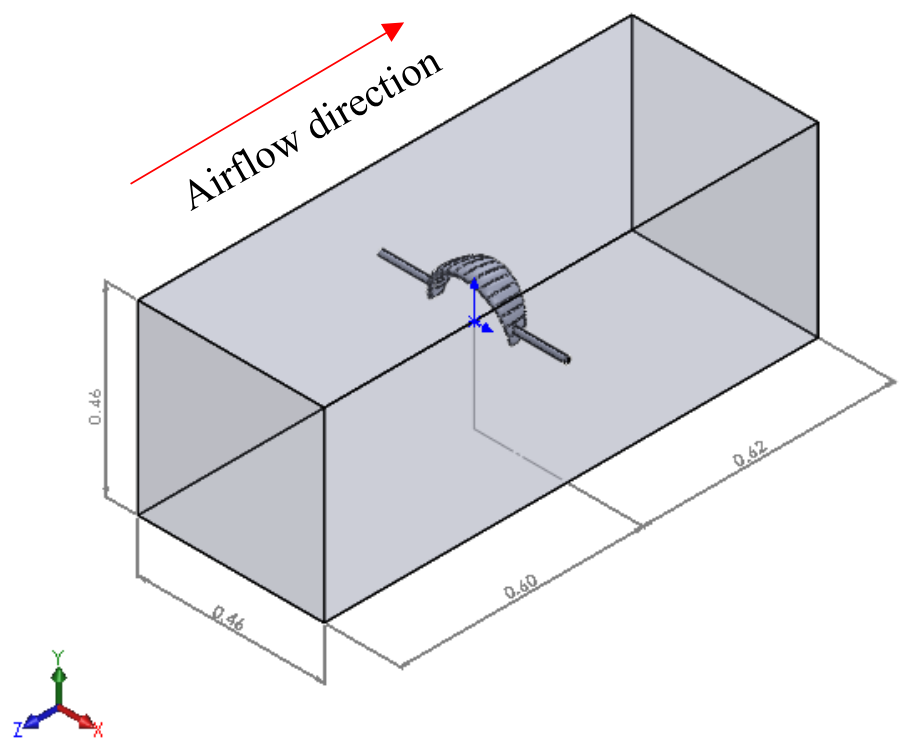


Figure 4 Mesh independence study

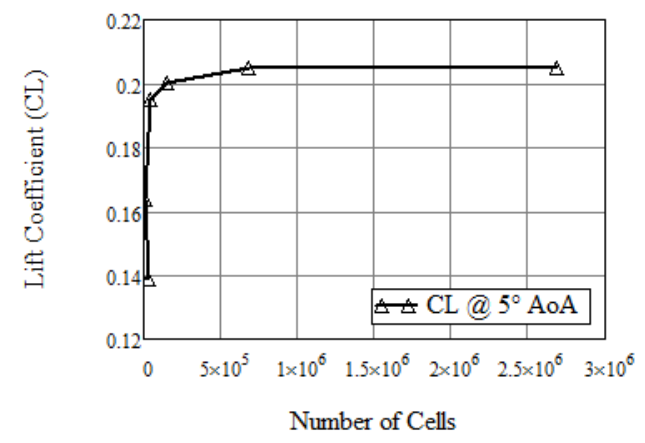

(a)

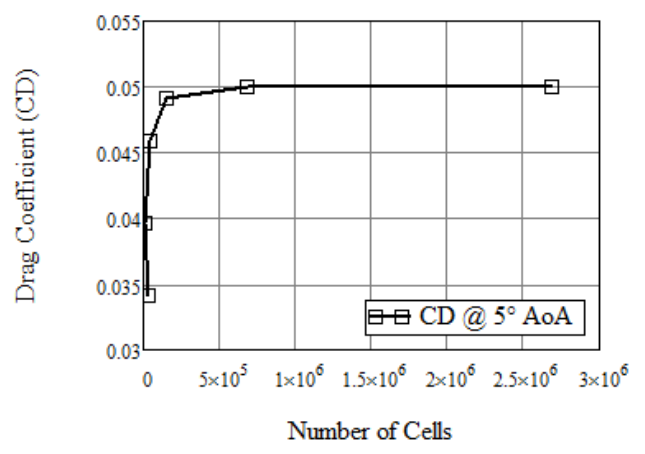

(b)

Figure 5 Comparison of Experimental and CFD results
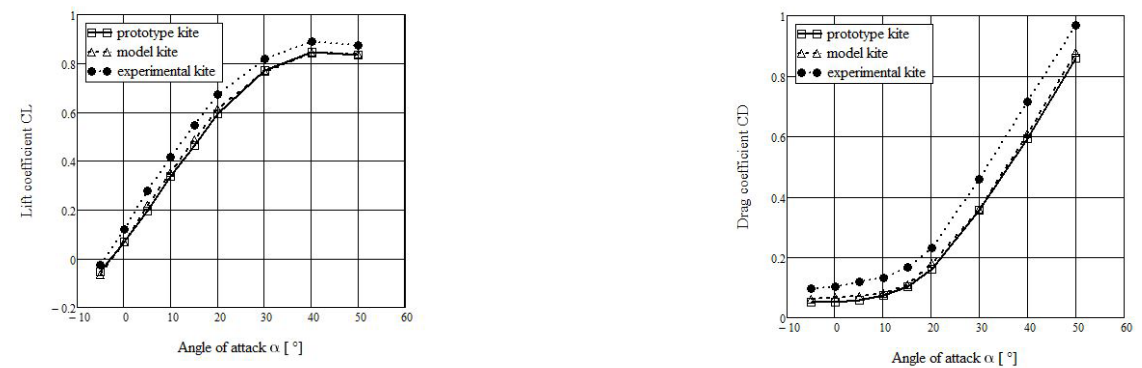

Figure 6 Velocity over kite- (a) AoA $0^{\circ}$ (b) AoA $40^{\circ}$ (see online version for colours)

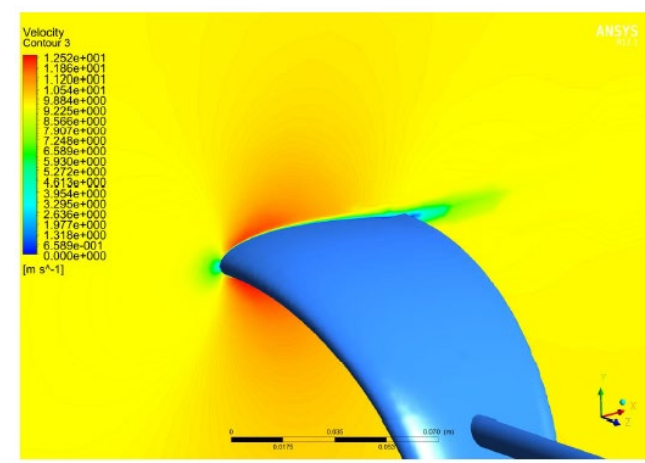

(a)

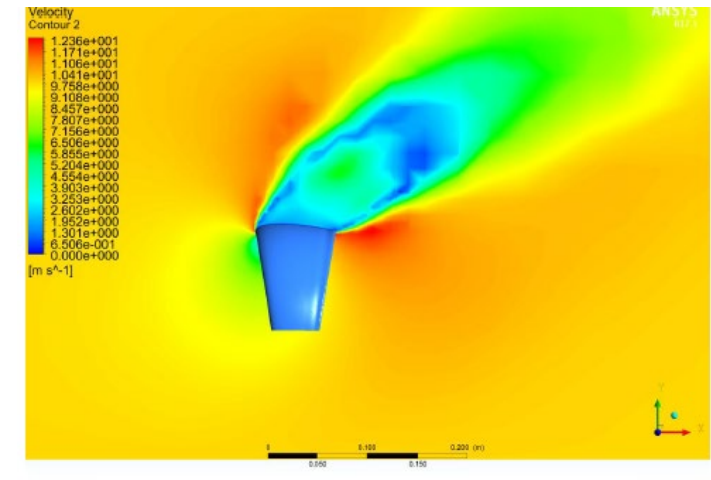

(b)

Figure 7 Vorticity region over kite- (a) AoA $0^{\circ}$ (b) AoA $40^{\circ}$ (see online version for colours)

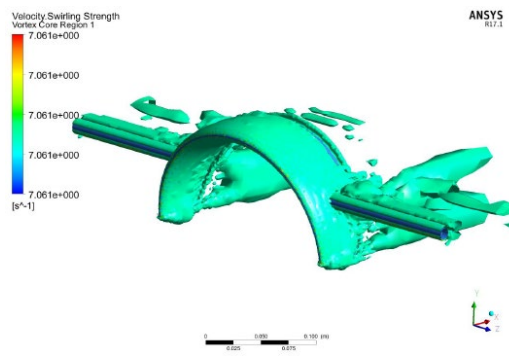

(a)

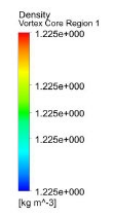

(b) 
Figure 8 Plot of drag and lift coefficients (see online version for colours)

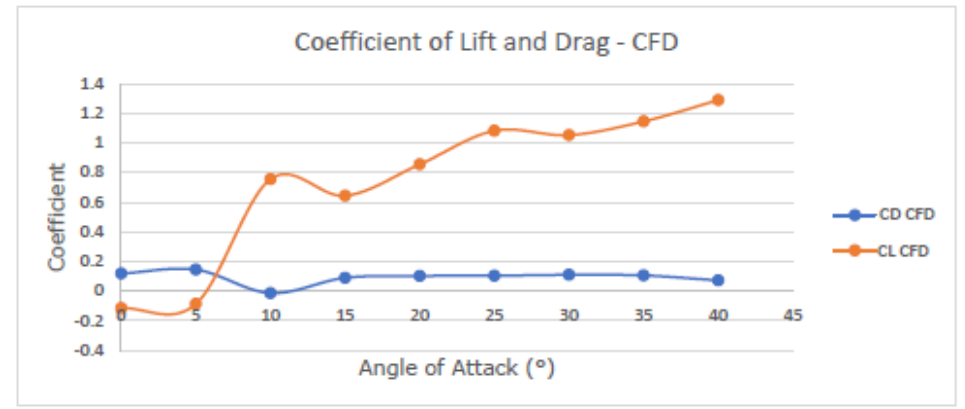

Figure 9 Comparison of velocity streamline plot for horizontal separation distance $400 \mathrm{~mm}$ and $200 \mathrm{~mm}$ (see online version for colours)

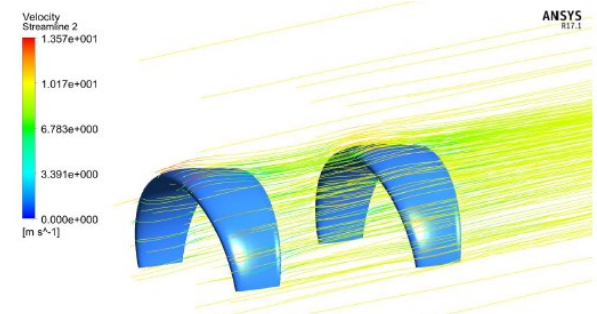

(a)

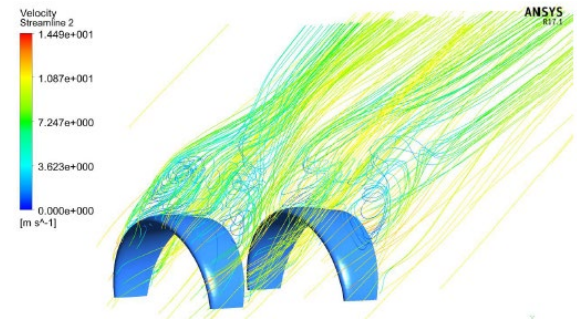

(b)

Figure 10 Lift and drag coefficients- (a) Powerkite 1 (b) Powerkite 2 (see online version for colours)

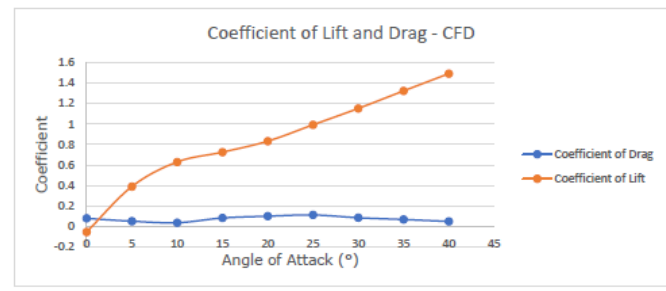

(a)

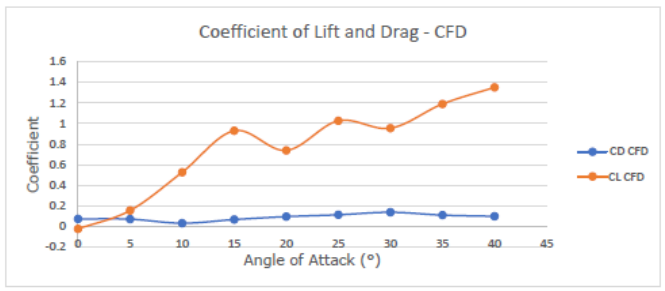

(b)

Figure 11 Pressure over wings (see online version for colours)

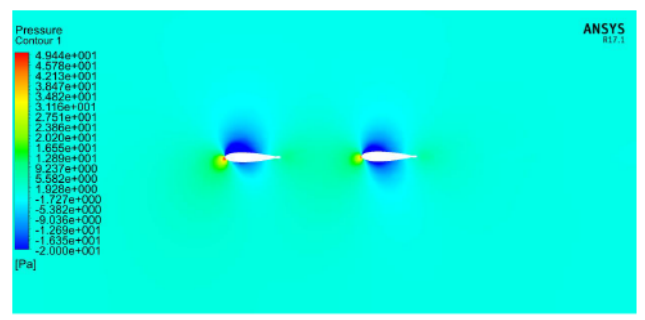

$-\ldots$

(a)

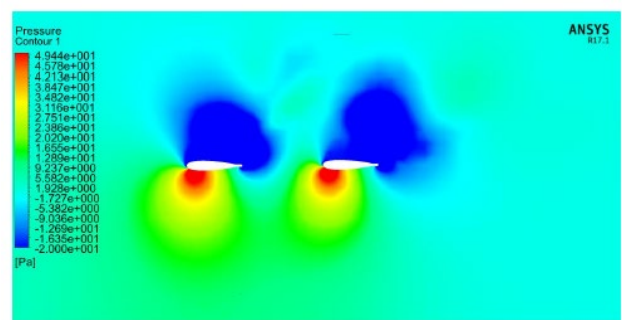

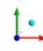

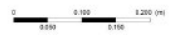

(b) 
Figure 12 Comparison of lift and drag coefficients for different (a) horizontal separation distances (b) vertical separation distances (see online version for colours)

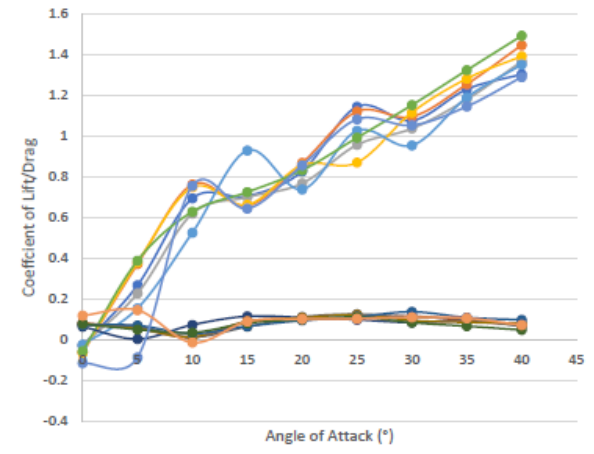

(a)
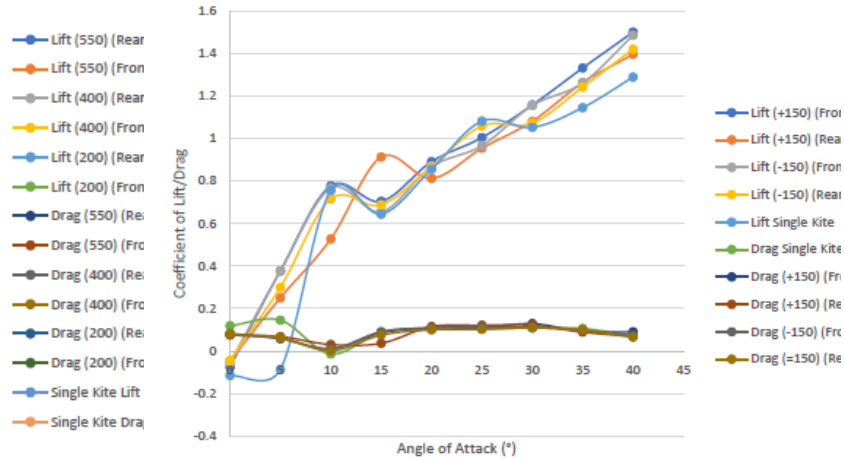

(b)

Figure 13 Drag and lift coefficients- (a) vertical displacement $150 \mathrm{~m}$ for leading kite (b) vertical displacement $150 \mathrm{~m}$ for trailing kite (c) vertical displacement $400 \mathrm{~m}$ for leading kite (d) vertical displacement $400 \mathrm{~m}$ for trailing kite (see online version for colours)

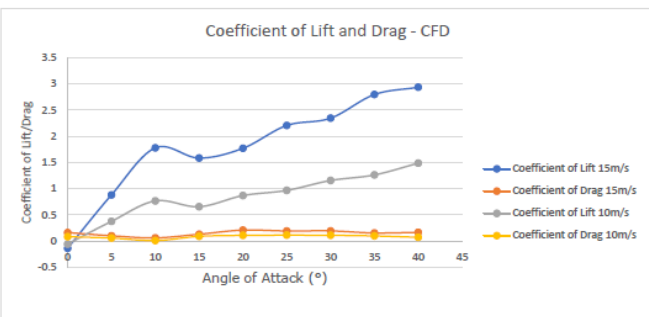

(a)

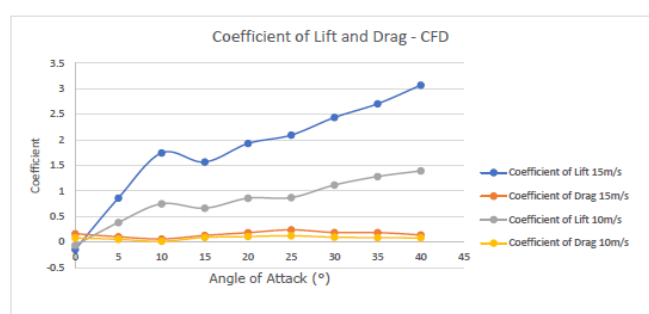

(c)

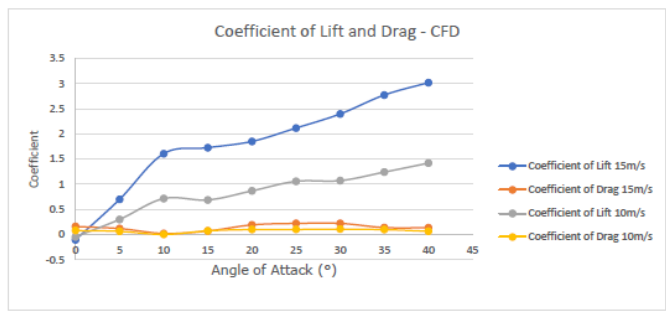

(b)

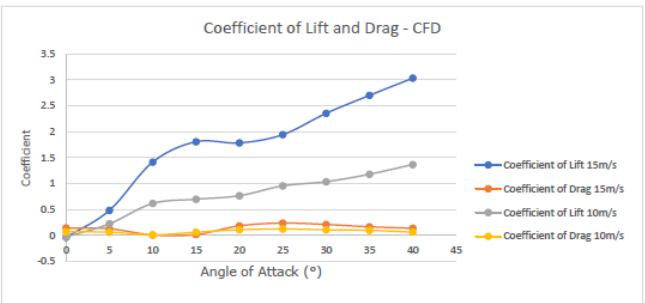

(d) 
Figure 14 Pressure over aerofoils at $150 \mathrm{~mm}$ vertical displacement (see online version for colours)

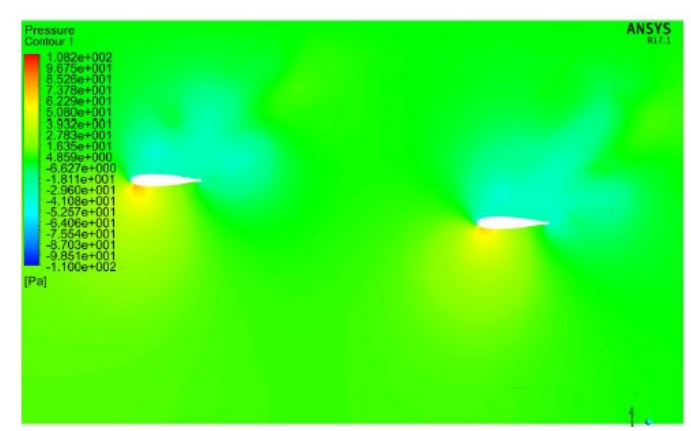

(a)

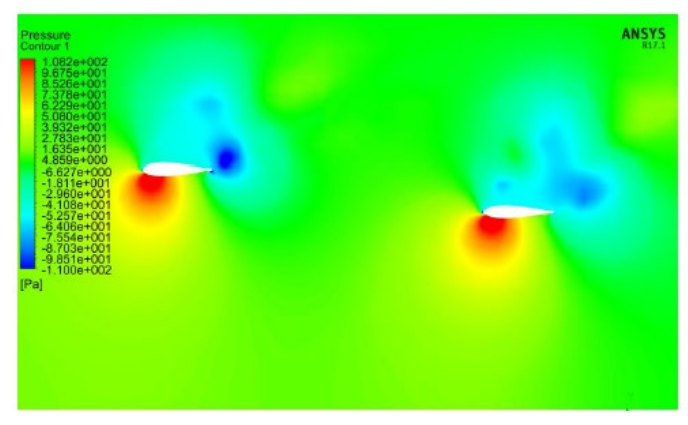

(b)

Figure 15 Comparison of lift and drag coefficients for smooth and rough aerofoil (see online version for colours)

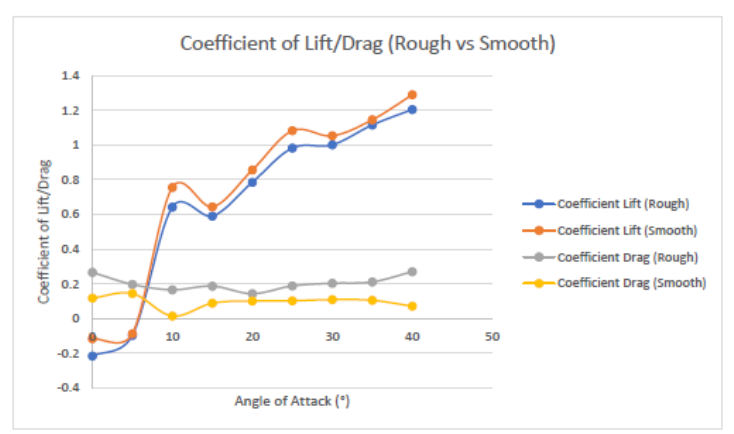

Figure 16 Vorticity and velocity contour plot in the rough surface kite (see online version for colours)

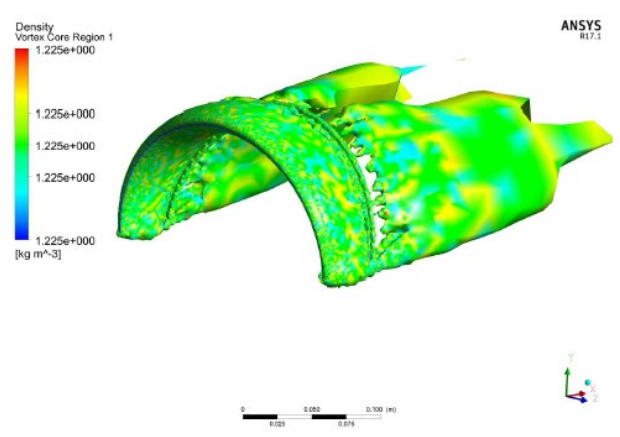

(a)

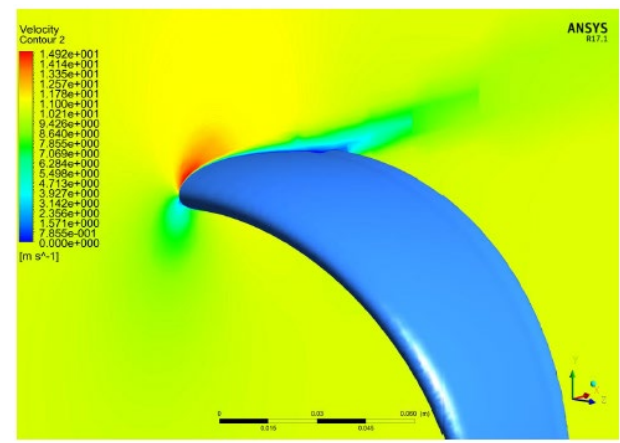

(b) 
Figure 17 (a) Billowed kite (b) Unbillowed kite

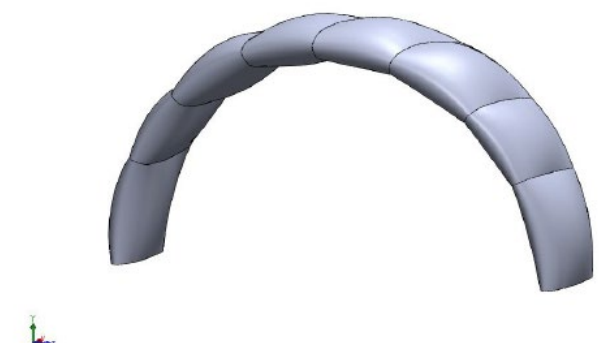

(a)

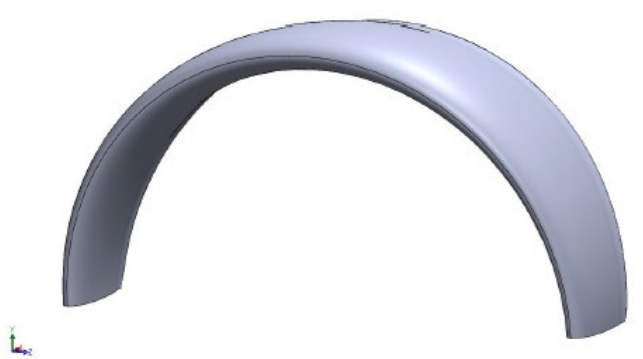

(b)

Figure 18 Drag and lift coefficient (a) Billowed kite (b) Unbillowed kite (see online version for colours)

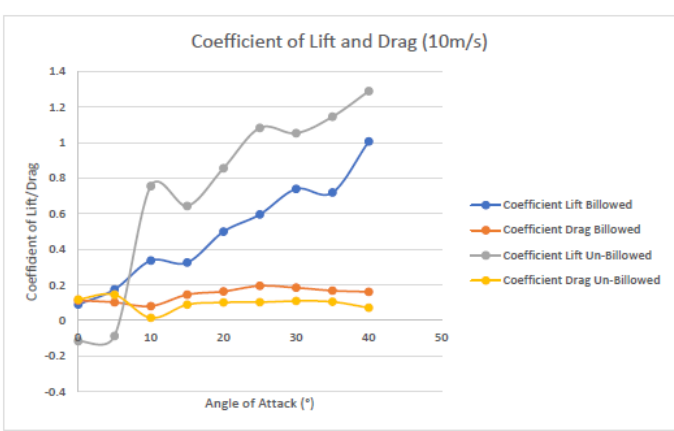

(a)

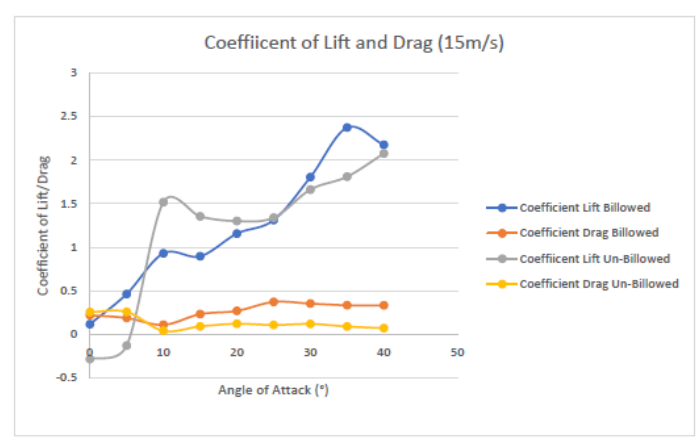

(b)

Figure 19 Type of aerofoil - (a) NACA 0018 (b) NACA 2412

(a)

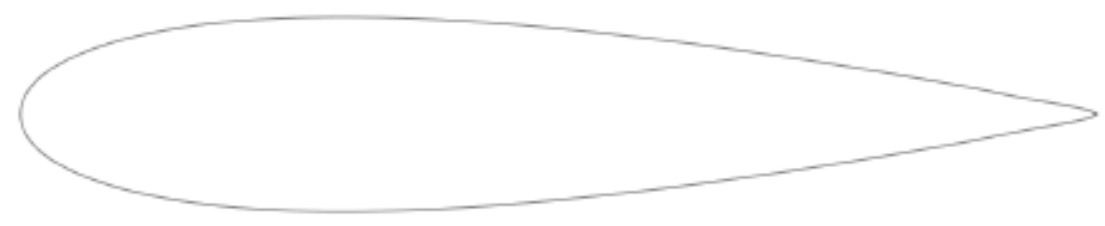

(b) 
Figure 20 Lift and drag comparison for NACA 2412 aerofoil with velocity (a) $10 \mathrm{~m} / \mathrm{s}$ (b) $15 \mathrm{~m} / \mathrm{s}$ (see online version for colours)

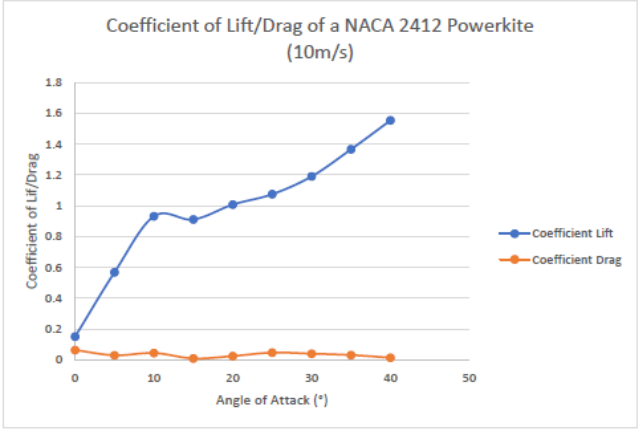

(a)

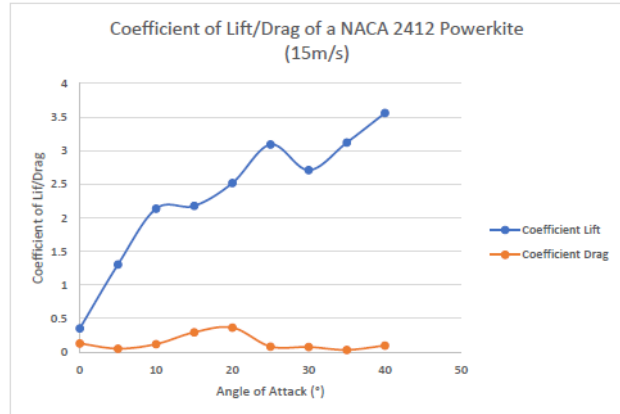

(b) 